PHOTOVOLTAICS

\title{
Voltage victory for kesterite solar cells
}

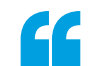

What will continue to drive studies of kesterite solar cells is the clear promise of...efficient, inexpensive, scalable and environmentally benign power generation
Although the efficiencies of solar cells with kesterite absorbers have increased since the fabrication of the first kesterite-based thin-film devices, the voltages of these solar cells have remained low. Now, writing in Nature Energy, Richard Haight and colleagues report kesterite solar cells that not only have near-record efficiencies but operate at voltages high enough to potentially be used in small, autonomous household electronics.

Kesterite, $\mathrm{Cu}_{2} \mathrm{ZnSn}\left(\mathrm{S}_{x} \mathrm{Se}_{1-x}\right)_{4}$, with a high $S$ content is known to increase the open-circuit voltage $\left(V_{\mathrm{OC}}\right)$ of solar cells based on these materials. However, this shift in the chalcogenide ratio also causes a decrease in the efficiency. Haight and colleagues have sought to overcome this performance issue by modifying the back contact of kesterite solar cells.

To make the solar cells, Haight and co-workers deposit a thin film of kesterite on a Mo-coated glass substrate and then anneal the coated system at high temperature with elemental S. Slow cooling of the system contributes to a further widening of the bandgap, possibly attributable to a decrease in the disorder of the $\mathrm{Cu}$ and $\mathrm{Zn}$ cations in the absorber. However, the annealing process has a detrimental effect on the material at and near the back contact. "To crystallize the thin film for optimal device performance, processing at temperatures of up to $\sim 600^{\circ} \mathrm{C}$ is required," explains Haight. "But at these temperatures, material phases, such as $\mathrm{Mo}(\mathrm{S}, \mathrm{Se})_{2}$, form at the back contact, which cause the overall resistance of the device to increase."

The breakthrough was the realization that further optimization of the devices would require room-temperature processing. "We developed a process whereby we first fabricate a high-functioning, fully characterized solar cell and then remove the back contact at room temperature through the mechanical process of exfoliation," says Haight. In the next step, a high-work-function $\mathrm{MoO}_{3} / \mathrm{Au}$ back contact is deposited, with the Au layer both protecting $\mathrm{MoO}_{3}$ from air exposure and reflecting long-wavelength light back into

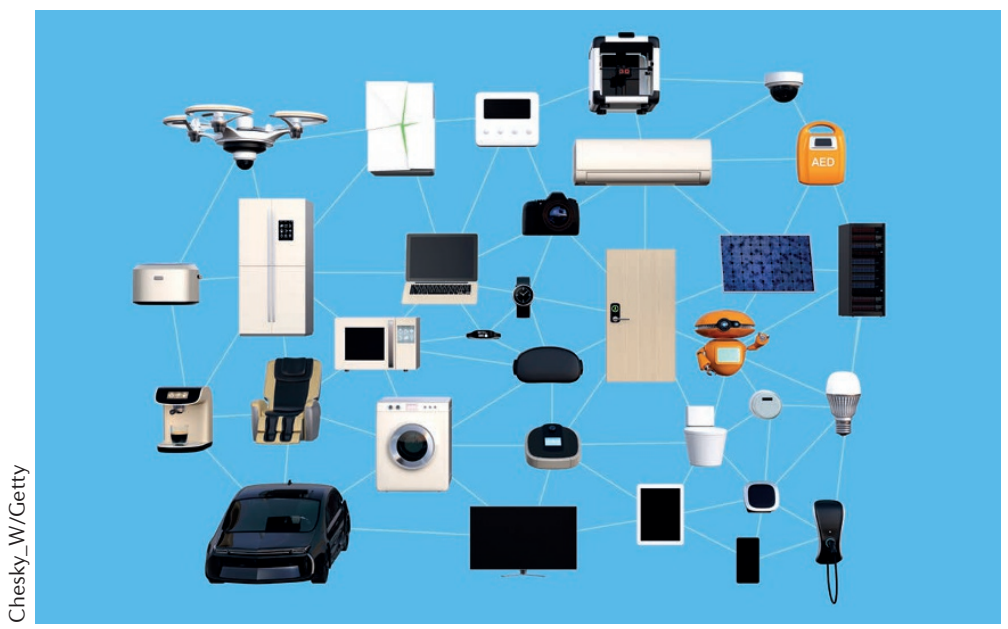

the absorber. The top-performing devices made using this approach showed $V_{\text {OC }}$ values of 669 and $628 \mathrm{mV}$ with associated efficiencies of $11.53 \%$ and $11.89 \%$, respectively, corresponding to an increase in $V_{\mathrm{OC}}$ of up to $160 \mathrm{mV}$ and efficiencies only $\sim 1 \%$ lower compared with the current 'record' kesterite solar cell.

The researchers connected nine of these high-voltage solar cells in series, achieving a $V_{\text {OC }}$ of $5.7 \mathrm{~V}$ under 1 Sun illumination. This series of solar cells also shows good performance at low light intensities ( $<10^{-3}$ Suns), achieving a $V_{\text {OC }}$ of $\sim 2 \mathrm{~V}$, which is enough voltage to charge a battery or run a low-power microprocessor. Moreover, the exposure of the back contact of the devices may allow for facile integration with, for example, batteries, microprocessors and sensors, providing the energy harvesting and storage required to enable a system of distributed electronics for the 'Internet of Things'. To demonstrate proof-of-concept, Haight and co-workers show that a serially connected device under 'desk lamp-level' illumination can power a microcontroller programmed to transmit a signal from a light-emitting diode.

The abundance and non-toxicity of kesterite make it a suitable material for use in our homes. However, the efficiencies of these solar cells will have to increase, which Haight suggests will be realized through mitigation of bulk defects (for example, by cation substitution) and careful engineering of the bandgap, as well as by improving the $\mathrm{p}-\mathrm{n}$ junction. "What will continue to drive studies of kesterite solar cells is the clear promise of, and hope for, efficient, inexpensive, scalable and environmentally benign power generation from the sun," he concludes.

Claire Ashworth 